\title{
Passing the boundary between the parity breaking medium and vacuum by vector particles
}

\section{Sergey Kolevatov*}

Saint Petersburg State University

E-mail: S.S.kolevatovegmail.com

\section{Alexander Andrianov}

Saint Petersburg State University

E-mail: andrianovdicc.ub.edu

\begin{abstract}
The problem of propagation of photons and massive vector mesons in the presence of Lorenz and CPT invariance violating medium is studied when the parity-odd medium is bounded by a hyperplane separating it from the vacuum. The solutions in both half-spaces are carefully discussed and in the case of space-like boundary stitched with help of the Bogolubov transformations. The presence of two different Fock vacua is shown. Classical solutions are also discussed. Effects of reflection from a boundary (up to the total one) are revealed when vector particles escape to vacuum or income from vacuum passing the boundary. Boundary effects under consideration are of certain importance for discovery of local parity violation in the collision of heavy ions and for registration of condensed axions in the dense astrophysical objects.
\end{abstract}

The XXI International Workshop High Energy Physics and Quantum Field Theory

June 23 - June 30, 2013

Saint Petersburg Area, Russia

\footnotetext{
* Speaker.
} 


\section{Introduction}

The interest to possible Lorentz and CPT Invariance Violation in the Standard Model was raised up in last 20 years after the seminal paper [U]. In that work the electrodynamics modified with additional Chern-Simons (CS) parity-odd lagrangian spanned on a constant CS four-vector was considered. From the analysis of the radiation of distant radio galaxies it was shown that there is no such violation on the Hubble scales. Nevertheless, the absence of experimental evidence of a tiny violation of Lorentz invariance and parity on scales comparable to the size of the universe, does not rule out such effects at the level of galaxies and stars. At this scales the spontaneous breaking of Lorenz invariance can occur as an effect of the medium after condensation of massless axion-like fields []. In addition, axions are one of the candidates for dark matter.

The recent results of the research group AMS-2 confirmed the data of earlier experiments PAMELA and Fermi-LAT , namely, the abnormally large number of high-energy positrons in cosmic rays and the absence of such an effect for antiprotons [B]]. Despite the fact that these results can be explained by astrophysical processes, the possibility of an abnormal yield of positrons due to the presence of the dark matter seems to be very interesting. In the presence of axion-like background, the photon can decay into a particle-antiparticle pair. In this case, the threshold of a pair is proportional to the squared mass of the produced particles [四]. This fact explains the abnormal number of positrons and the absence of the effect for antiprotons, since $\frac{m_{e}^{2}}{m_{p}^{2}} \sim 10^{-6}$.

Another interesting area for observation of parity breaking is the heavy ion physics. Recently several experiments in heavy ion collisions have indicated an abnormal yield of lepton pairs of invariant mass $<1 \mathrm{GeV}$ in the region of small rapidities and moderate transversal momenta [\$] . This phenomenon is stronger for central collisions with moderate transversal momenta of dileptons. It happens both for $e^{+} e^{-}$and dimuon pairs and a possible explanation of this enhancement is outlined in [6]. It was conjectured that the effect may be a manifestation of local parity breaking (LPB) in colliding nuclei due to generation of pseudoscalar, isosinglet or neutral isotriplet, classical background whose magnitude depends on the dynamics of the collision.

In the occurrences of axion-like background in astrophysics or heavy ion physics the existence of a boundary between the parity-odd medium and the vacuum is quite essential. For star condensed axions there is evidently a boundary where axion background disappears and photons distorted by it escape to vacuum. However, not all the photons penetrate the boundary and partially a reflection arises. As well in heavy ion collisions the outcome of the photon/vector meson decays, say, into lepton pairs generated by slowly decreasing pseudoscalar background inside of the fireball can be normally registered in vacuum or after freeze-out when dilepton pairs are outside of the parity breaking medium.

\section{Carroll-Field-Jackiw model with the boundary}

We start from the Lagrange density which describes the propagation of a vector field in the presence of a pseudoscalar axion-like background,

$$
\begin{aligned}
\mathscr{L} & =-\frac{1}{4} F^{\alpha \beta}(x) F_{\alpha \beta}(x)-\frac{1}{4} F^{\mu v}(x) \widetilde{F}_{\mu v}(x) a_{c \ell}(x) \\
& +\frac{1}{2} m^{2} A_{v}(x) A^{v}(x)+A^{\mu}(x) \partial_{\mu} B(x)+\frac{1}{2} \varkappa B^{2}(x),
\end{aligned}
$$


where $A_{\mu}$ and $a_{c \ell}$ stand for the vector and background pseudoscalar fields respectively, $\widetilde{F}^{\mu \nu}=$ $\frac{1}{2} \varepsilon^{\mu v \rho \sigma} F_{\rho \sigma}$ is the dual field strength, while $B$ is the auxiliary Stückelberg scalar field with $\varkappa \in \mathbb{R}$. The positive dimensionless coupling $g>0$ and the mass parameter $M \gg m$ do specify the intensity and the scale of the pseudoscalar-vector interaction. Notice that the Proca mass term was included for the vector field. It is required to account for the strong interaction effects in heavy ion collisions supported by massive vector mesons $(\rho, \omega, \ldots)$ [ [ ] in addition to photons. The auxiliary Stückelberg lagrangian, which further violates gauge invariance beyond the mass term for the vector field, has been introduced to provide - just owing to the renowned Stuickelberg trick - the simultaneous occurrences of power counting renormalizability and perturbative unitarity for a general interacting theory. Moreover, its presence allows for a smooth massless limit of the quantized vector field [?].

Let us consider a slowly varying classical pseudoscalar background of the kind,

$$
a_{c \ell}(x)=\zeta_{\lambda} x^{\lambda} \theta(-\zeta \cdot x)
$$

where $\theta(\cdot)$ is a Heaviside step distribution, in which a fixed constant four vector $\zeta^{\mu}$ with dimension of a mass has been introduced, in a way to violate Lorentz and CPT invariances in the Minkowski half space $\zeta \cdot x<0$. If we now insert the specific form (2.2) of the pseudoscalar background in the pseudoscalar-vector coupling lagrangian we find the field equations,

$$
\left\{\begin{array}{cc}
\partial_{\lambda} F^{\lambda v}+m^{2} A^{v}+\zeta_{\alpha} \widetilde{F}^{\alpha v}+\partial^{v} B=0 & \text { for } \zeta \cdot x<0 \\
\partial_{\lambda} F^{\lambda v}+m^{2} A^{v}+\partial^{v} B=0 & \text { for } \zeta \cdot x>0 \\
\partial_{v} A^{v}=\varkappa B &
\end{array}\right.
$$

After contraction of the first pair of the above set of field equations with $\partial_{v}$ we find, that the auxiliary Stückelberg field is always a decoupled unphysical real scalar field, which is never affected by the pseudoscalar classical background $\forall \varkappa \in \mathbb{R}$. From now on we shall select the simplest choice $\varkappa=1$ that leads to the Klein-Gordon equation for the auxiliary field and the next equations for vector field,

$$
\left\{\begin{array}{cc}
\square A^{v}(x)+m^{2} A^{v}(x)=\varepsilon^{v \alpha \rho \sigma} \zeta_{\alpha} \partial_{\rho} A_{\sigma}(x) & \zeta \cdot x<0 ; \\
\square A^{v}(x)+m^{2} A^{v}(x)=0 & \zeta \cdot x>0 .
\end{array}\right.
$$

The general solution of these field equations is well-known Proca-Stückelberg vector field for $\zeta$. $x>0$ and Maxwell-Chern-Simons massive vector field for $\zeta \cdot x<0$, this solutions were discussed in details in $[\mathbb{U}]$. Let us discuss here the case of a spatial Chern-Simons vector $\zeta_{\mu}=\left(0,-\zeta_{x}, 0,0\right)$, and set such objects: $\hat{k}=\left(\omega, k_{2}, k_{3}\right), \hat{x}=\left(x_{0}, x_{2}, x_{3}\right): \hat{k} \cdot \hat{x}=-\omega x_{0}+k_{2} x_{2}+k_{3} x_{3}$. We can write solution in form:

$$
\begin{aligned}
& A_{\mathrm{PS}}^{\mu}(x)=\int \mathrm{d} \hat{k} \boldsymbol{\theta}\left(\omega^{2}-k_{\perp}^{2}-m^{2}\right) \sum_{r=1}^{3}\left[\mathbf{a}_{\hat{k}, r} u_{\hat{k}, r}^{\mu}(x)+\mathbf{a}_{\hat{k}, r}^{\dagger} u_{\hat{k}, r}^{\mu *}(x)\right], \quad \partial_{\mu} A_{\mathrm{PS}}^{\mu}(x)=0, \\
& u_{\hat{k}, r}^{v}(x)=\left[(2 \pi)^{3} 2 k_{10}\right]^{-1 / 2} e_{r}^{v}(\hat{k}) \exp \left\{i k_{10} x_{1}+i \hat{k} \cdot \hat{x}\right\} \quad(r=1,2,3)
\end{aligned}
$$

for $x_{1}>0$, where the creation destruction operators fulfill the canonical commutation relations $\left[\mathbf{a}_{\hat{k}, r}, \mathbf{a}_{\hat{k}^{\prime}, s}^{\dagger}\right]=\delta\left(\hat{k}-\hat{k}^{\prime}\right) \delta_{r s}$. The three linear polarization real vectors do satisfy the orthonormality and closure relations on the mass shell $k^{2}=m^{2}$ : namely,

$$
k_{\mu} e_{r}^{\mu}(\hat{k})=0 \quad-g_{\mu v} e_{r}^{\mu}(\hat{k}) e_{s}^{v}(\hat{k})=\delta_{r s} \quad \sum_{r=1}^{3} e_{r}^{\mu}(\hat{k}) e_{r}^{v}(\hat{k})=-g^{\mu v}+\frac{k^{\mu} k^{v}}{m^{2}}
$$


In order to write solution for $x_{1}<0$, we should find dispersion laws. For the spatial CS vector we get,

$$
\left\{\begin{array}{c}
k_{1 L}=k_{10}=\sqrt{\omega^{2}-m^{2}-k_{\perp}^{2}} \\
k_{1-}=\sqrt{\omega^{2}-m^{2}-k_{\perp}^{2}+\zeta_{x} \sqrt{\omega^{2}-k_{\perp}^{2}}} \\
k_{1+}=\sqrt{\omega^{2}-m^{2}-k_{\perp}^{2}-\zeta_{x} \sqrt{\omega^{2}-k_{\perp}^{2}}}
\end{array}\right.
$$

and the solution is,

$$
\begin{aligned}
& A_{\mathrm{CS}}^{v}(x)=\int \mathrm{d} \hat{k} \theta\left(\omega^{2}-k_{\perp}^{2}-m^{2}\right) \sum_{A}\left[c_{\hat{k}, A} v_{\hat{k} A}^{v}(x)+c_{\hat{k}, A}^{\dagger} v_{\hat{k} A}^{v *}(x)\right], \quad A \in\{L,+,-\}, \\
& v_{\hat{k} A}^{v}(x)=\left[(2 \pi)^{3} 2 k_{1 A}\right]^{-\frac{1}{2}} \varepsilon_{A}^{v}(k) \exp \left\{i k_{10} x_{1}+i \hat{k} \cdot \hat{x}\right\}
\end{aligned}
$$

where $c_{\hat{k}, A}^{\dagger}, c_{\hat{k}, A}$ are creation and destruction operators with canonical commutation relations, $\varepsilon_{A}^{v}(k)$ are polarization vectors, which are carefully discussed e.g. in [ [8].

The Proca-Stückelberg vector field and the Maxwell-Chern-Simons massive vector field face one another at the hyperplane $\zeta \cdot x=0$. Hence locality of the quantized wave fields does require equality on the surface separating the classical pseudoscalar background from the vacuum: namely,

$$
\left[A_{\mathrm{PS}}^{\mu}(x)-A_{\mathrm{CS}}^{\mu}(x)\right]_{x_{1}=0}=0
$$

Now, we suggest that $v_{\hat{k}, A}^{v}(\hat{x})$ and $u_{\hat{k}, s}^{v}(\hat{x})$ are related by Bogolyubov transformation,

$$
v_{\hat{k}, A}^{v}(\hat{x})=\sum_{s=1}^{3}\left[\alpha_{s A}(\hat{k}) u_{\hat{k}, s}^{v}(\hat{x})-\beta_{s A}(\hat{k}) u_{\hat{k}, s}^{v *}(\hat{x})\right]
$$

Using the boundary condition (R.9) and taking the Bogolyubov transformation (R.JU) into account, we can write the operator equalities,

$$
\begin{array}{r}
\mathbf{a}_{\hat{k}, r}=\sum_{A= \pm, L}\left[\alpha_{r A}(\hat{k}) c_{\hat{k}, A}-\beta_{r A}^{*}(\hat{k}) c_{\hat{k}, A}^{\dagger}\right] \\
c_{\hat{k}, A}=\sum_{r=1}^{3}\left[\alpha_{A r}^{*}(\hat{k}) \mathbf{a}_{\hat{k}, r}+\beta_{A r}^{*}(\hat{k}) \mathbf{a}_{\hat{k}, r}^{\dagger}\right]
\end{array}
$$

There are two different Fock vacua: namely,

$$
\mathbf{a}_{\hat{k}, r}|0\rangle=0 \quad c_{\hat{k}, A}|\Omega\rangle=0
$$

\section{Vacuum as a squeezed state}

In the previous section we have found that in our model there are two different Fock vacua: $|0\rangle,|\Omega\rangle$. It is natural to assume that there should be a relation between this two states. We will take the most general form of this relation,

$$
|0\rangle_{\hat{k}}=\sum_{p, m, l=0}^{\infty} f_{p m l} \frac{\left(c_{\hat{k},+}^{\dagger}\right)^{p}\left(c_{\hat{k},-}^{\dagger}\right)^{m}\left(c_{\hat{k}, L}^{\dagger}\right)^{l}}{\sqrt{p ! m ! l ! !}}|\Omega\rangle_{\hat{k}},
$$


where

$$
|\Omega\rangle=\prod_{\hat{k}}\left(|\Omega\rangle_{\hat{k}}\right) \quad|0\rangle=\prod_{\hat{k}}\left(|0\rangle_{\hat{k}}\right),
$$

and the product is taken over all possible values of $\hat{k}$. Later we will consider the continuum limit. In order to find coefficients $f_{k m l}$, let's insert (‥JI) in the next identity,

$$
\mathbf{a}_{\hat{k}, r}|0\rangle_{\hat{k}}=0
$$

It gives us the relation between the vacua,

$$
|0\rangle_{\hat{k}}=\sum_{p, m, l=0}^{\infty} \frac{1}{(2 p) ! !(2 m) ! !(2 l) ! !}\left(\frac{\beta_{r+}^{*}(\hat{k})}{\alpha_{r+}(\hat{k})}\right)^{p}\left(\frac{\beta_{r-}^{*}(\hat{k})}{\alpha_{r-}(\hat{k})}\right)^{m}\left(\frac{\beta_{r L}^{*}(\hat{k})}{\alpha_{r L}(\hat{k})}\right)^{l}\left(c_{\hat{k},+}^{\dagger}\right)^{2 p}\left(c_{\hat{k},-}^{\dagger}\right)^{2 m}\left(c_{\hat{k}, L}^{\dagger}\right)^{2 l}|\Omega\rangle_{\hat{k}}
$$

Easy to see that the expression above is a Taylor series of an exponent.

$$
|0\rangle_{\hat{k}}=\exp \left[\frac{\beta_{r+}^{*}(\hat{k})}{2 \alpha_{r+}(\hat{k})}\left(c_{\hat{k},+}^{\dagger}\right)^{2}+\frac{\beta_{r-}^{*}(\hat{k})}{2 \alpha_{r-}(\hat{k})}\left(c_{\hat{k},-}^{\dagger}\right)^{2}+\frac{\beta_{r L}^{*}(\hat{k})}{2 \alpha_{r L}(\hat{k})}\left(c_{\hat{k}, L}^{\dagger}\right)^{2}\right]|\Omega\rangle_{\hat{k}}
$$

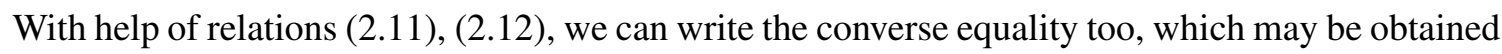
by the next changes $\alpha_{r A}(\hat{k}) \rightarrow \alpha_{A r}^{*}(\hat{k}) ;\left(-\beta_{r A}^{*}(\hat{k})\right) \rightarrow \beta_{A r}^{*}(\hat{k}) ; c_{\hat{k}, A} \rightarrow \mathbf{a}_{\hat{k}, r}$. Thus, quite analogous to the previous case,

$$
|\Omega\rangle_{\hat{k}}=\exp \left[\frac{-\beta_{A 1}^{*}(\hat{k})}{2 \alpha_{A 1}^{*}(\hat{k})}\left(\mathbf{a}_{\hat{k}, 1}^{\dagger}\right)^{2}+\frac{-\beta_{A 2}^{*}(\hat{k})}{2 \alpha_{A 2}^{*}(\hat{k})}\left(\mathbf{a}_{\hat{k}, 2}^{\dagger}\right)^{2}+\frac{-\beta_{A 3}^{*}(\hat{k})}{2 \alpha_{A 3}^{*}(\hat{k})}\left(\mathbf{a}_{\hat{k}, 3}^{\dagger}\right)^{2}\right]|0\rangle_{\hat{k}},
$$

and for both vacua we obtain next relations,

$$
\begin{array}{r}
|0\rangle=\exp \left[\int\left(\sum_{A= \pm, L} \frac{\beta_{r A}^{*}(\hat{k})}{2 \alpha_{r A}(\hat{k})}\left(c_{\hat{k}, A}^{\dagger}\right)^{2} \theta\left(k_{1 A}^{2}(\hat{k})\right)\right) d \hat{k}\right]|\Omega\rangle \\
|\Omega\rangle=\exp \left[\int \theta\left(\omega^{2}-m^{2}-k_{\perp}^{2}\right)\left(\sum_{r=1,2,3} \frac{-\beta_{A r}^{*}(\hat{k})}{2 \alpha_{A r}^{*}(\hat{k})}\left(\mathbf{a}_{\hat{k}, r}^{\dagger}\right)^{2}\right) d \hat{k}\right]|0\rangle .
\end{array}
$$

Pay attention to the $\theta$-functions, which stand here to integrate over physical values of $\hat{k}$. Notice that in our model both of the Fock vacua are real, but every one in its region. That's why we may say that vacuum state in the presence of CS field can be described in terms of Proca-Stückelberg vacuum and vice versa.

\section{Passing the boundary}

We consider classical solutions of field equations (2.4). Using the matching condition on the boundary (R.9) for spatial CS vector one can find the reflection coefficient as a function of the invariant mass of the outgoing particle [Q],

$$
k_{r e f}=\frac{\left|\sqrt{\frac{\left(M^{2}-m^{2}\right)^{2}}{\zeta^{2}}-M^{2}}-\sqrt{\frac{\left(M^{2}-m^{2}\right)^{2}}{\zeta^{2}}-m^{2}}\right|}{\left|\sqrt{\frac{\left(M^{2}-m^{2}\right)^{2}}{\zeta^{2}}-M^{2}}+\sqrt{\frac{\left(M^{2}-m^{2}\right)^{2}}{\zeta^{2}}-m^{2}}\right|}
$$


This expression is quite useful since in the case of the dilepton decay of the particle in parity odd medium, it is an invariant mass of the lepton pair, which is the observable quantity. Such a dependence on the invariant mass allows us to plot the $k_{r e f}\left(M^{2}\right)$. Let us consider the case of vector mesons. We choose the mass for them $780 \mathrm{MeV}$, because our goal is to describe a behavior of the particles on the boundary, and masses of $\rho$ and $\omega$ mesons are close to this value. In the case of vector mesons, reflectivity of astronomical objects are not as interesting as a possibility to observe some effects associated with the parity violation in the collisions of heavy ions. That's why for vector mesons we assume [目] $\zeta=300 \mathrm{MeV}$. The dependence of reflection coefficient on the invariant mass is shown in Fig.1.

We can see in the graph that for vector mesons with negative polarization there is a range of values of the invariant mass, where reflection coefficient is equal to 1, i.e. vector mesons can't cross the boundary. It happens for the next values of invariant mass, $\sqrt{m^{2}-m \zeta}<M<\sqrt{m^{2}+\frac{\zeta^{2}}{4}}-\frac{\zeta}{2}$, the upper limit is just a kinematical boundary for (-) polarization.

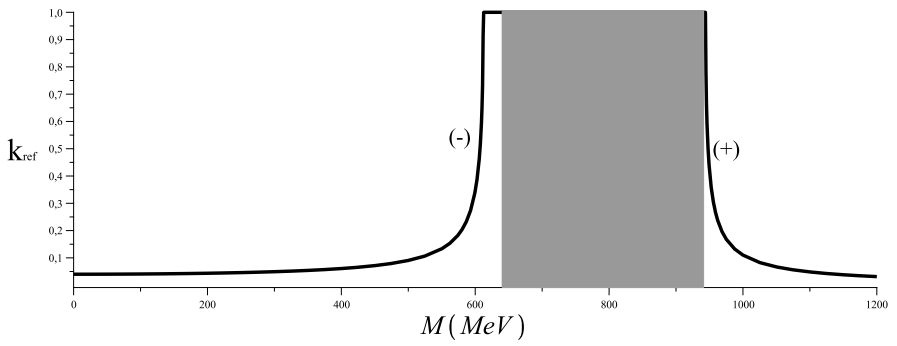

Figure 1: The reflection coefficient from the boundary for vector mesons escaping the parity odd region. The kinematically forbidden range of values of the invariant mass is shaded. $\zeta=300 \mathrm{MeV}$ is taken.

One can find the graph for the photon escaping from the parity breaking region in paper [Q]. In this work also a case of the entrance to the parity odd medium is considered. This case is quite important for photons, in fact the reflection of a light from the boundary of axion star or axion background near dense stars may be an observable process. And it was shown, that the reflection from the boundary of such object will give additional phase shift to circular polarizations of incoming photons.

It is also interesting to consider time-like CS vector. This case may be useful for a description of processes occurring in heavy ion collisions because it helps to understand what happens with particles inside the fireball. The parity-breaking mechanism, which may occur in these processes was described in [6]. In this paper a possible explanation for the abnormal yield of the lepton pairs in PHENIX/ CERES/ NA60 experiments was given. In that work CS vector had only one non-zero component, $\zeta_{\mu}=(\zeta, 0,0,0)$. However, in case of collisions of heavy ions, fireball has a finite size. In this work we aim to describe boundary effects, so let's modify CS vector and use the simplified model with two half-spaces,

$$
\zeta_{\mu}=\left(\zeta \theta\left(-x_{1}\right),-\zeta t \delta\left(x_{1}\right), 0,0\right)
$$

One can see in this expression a singularity on the boundary, which helps us conserve gauge invariance and does not contribute to any reflection effects. Just as for spatial CS vector we find the reflection coefficient,

$$
k_{r e f}=\frac{\left|\sqrt{\frac{\left(M^{2}-m^{2}\right)^{2}}{\zeta^{2}}-k_{\perp}^{2}}-\sqrt{\frac{\left(M^{2}-m^{2}\right)^{2}}{\zeta^{2}}+\left(M^{2}-m^{2}\right)-k_{\perp}^{2}}\right|}{\left|\sqrt{\frac{\left(M^{2}-m^{2}\right)^{2}}{\zeta^{2}}-k_{\perp}^{2}}+\sqrt{\frac{\left(M^{2}-m^{2}\right)^{2}}{\zeta^{2}}+\left(M^{2}-m^{2}\right)-k_{\perp}^{2}}\right|},
$$

which depends on $M$ and $k_{\perp}$. We present here a graph of this dependence for $\omega$-meson. 
In Fig. 2 one can see, that for the some values of transversal momentum and invariant mass the vector meson created in the fireball cannot leave it, because it undergoes a total reflection at these values. .

\section{Conclusions}

Main results of this work were obtained for the spatial CS vector. For this case the relation between two different Fock vacua was obtained, and it was shown, that each of them can be presented as a squeezed state in terms of the other one. The relations are presented which are suitable to calculate the reflection of incoming particles from the parity-odd domain.

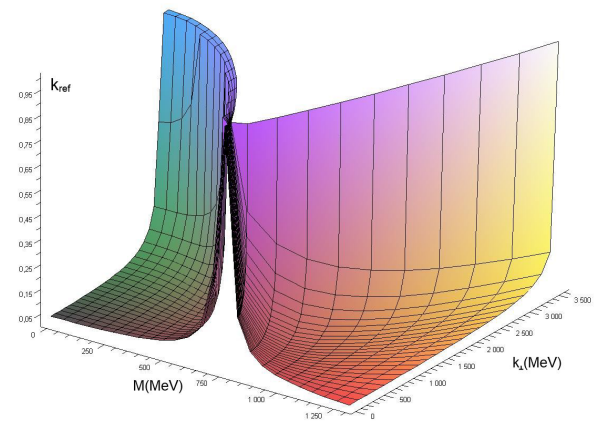

Figure 2: The refection coefficient for vector mesons escaping from the parity odd region. Time-like CS vector. $\zeta=300 \mathrm{MeV}$ is taken.

In particular, it was shown that transversal polarizations undergo strong reflection (up to the total internal one) at certain values of frequency. In addition, for the spatial CS vector it was revealed that during the irradiation of parity odd medium by photons an additional rotation of circular polarizations may take place upon reflection from the boundary. The analogous relations are found for the time-like CS vector with a spatial boundary, when the reflection coefficient depends on the transversal momentum of moving particle.

Acknowledgments. This investigation was supported in part by the Russian Foundation for Basic Research (Grant 13-02-00127), Saint Petersburg State University (Project 11.38.660.2013) and the non-profit Dynasty Foundation(S.S.K.).

\section{References}

[1] S. M. Carroll, G. B. Field and R. Jackiw, Phys. Rev. D 41, 1231 (1990).

[2] A. A. Andrianov and R. Soldati, Phys. Rev. D 51, 5961 (1995) [hep-th/ 9405147 ].

[3] M. Ibe, S. Iwamoto, S. Matsumoto, T. Moroi and N. Yokozaki, JHEP 1308 (2013) 029 [1304 . 1483 [hep-ph] ].

[4] A. A. Andrianov, D. Espriu, P. Giacconi and R. Soldati, JHEP 0909 (2009) 057 [0 907.3709 [hep-ph] ].

[5] A. Adare et al. [PHENIX Collaboration], Phys. Rev. C 81 (2010) 034911 [0912. 0244 [nucl-ex]].

[6] A. A. Andrianov, V. A. Andrianov, D. Espriu and X. Planells, Dilepton excess from local parity breaking in baryon matter, Phys. Lett. B $\mathbf{7 1 0}$ (2012) 230 [1201.3485 [hep-ph] ].

[7] A. A. Andrianov, S. S. Kolevatov and R. Soldati, JHEP 1111 (2011) 007 [1109. 3440 [hep-ph ] ].

[8] J. Alfaro, A. A. Andrianov, M. Cambiaso, P. Giacconi and R. Soldati, Int. J. Mod. Phys. A 25 (2010) $3271[0904.3557$ [hep-th] ];

[9] A. A. Andrianov and S. S. Kolevatov, Theor. Math. Phys. 175 (2013) 744 [Teor. Mat. Fiz. 175 (2013) 357] [1212.5723 [hep-ph]]. 